\title{
A Framework for Public Health in the United States
}

\author{
Jonathan E. Fielding, MD, MPH, MBA, ${ }^{1,2,3}$ \\ Steven Teutsch, MD, MPH, ${ }^{1}$ \\ Lester Breslow, $\mathrm{MD}^{2}$
}

\begin{abstract}
As in other countries, public heath in the United States continues to evolve to ensure healthy families and communities as well as individuals. Great achievements in the control of infectious and chronic disease and injuries will need to be sustained while we face new challenges, including providing universal access to high quality healthcare as well as addressing the underlying behavioral risk factors and the social, physical and environmental determinants of health. Meeting these challenges will require strengthening the governmental and non-governmental public health systems and working closely with other sectors.
\end{abstract}

Key words: Public Health, social and physical determinants, health United States

\section{INTRODUCTION}

The $19^{\text {th }}$ and $20^{\text {th }}$ centuries saw enormous changes in health and public health practice. The sanitary movement curbed the most burdensome infectious diseases by eliminating some human disease vectors, developing effective sewage systems, and requiring pasteurized milk, safer foods and safe drinking water, while enforcing tougher safety standards for homes and worksites. Life expectancy grew by an unprecedented average of 0.3 years annually throughout the $20^{\text {th }}$ century, the equivalent of three additional years of life every decade. Food and leisure became plentiful, and chronic diseases became the new frontier of public health.

\footnotetext{
${ }^{1}$ Los Angeles County Department of Public Health.

${ }^{2}$ UCLA School of Public Health.

${ }^{3}$ UCLA School of Medicine.
}

Correspondence: Jonathan Fielding at email jfieldin@ucla.edu and jfielding@ph.lacounty. gov; 313 N. Figueroa St., Rm. 804, Los Angeles, CA 90012 USA. 
In the last half of the 20th century, the greatest improvements in life expectancy were among those 65 and older. Reductions in smoking along with control of hypertension and other risk factors gradually helped reduce epidemic rates of heart disease and some cancers. However, more visibly, the 20th century also saw the growth of biomedical science and greater understanding of the pathophysiology of disease. That understanding led to an explosion of diagnostic and therapeutic technologies that could be delivered to individuals. Over time, the biomedical model with its focus on the individual became the dominant paradigm of health in the United States. It was accompanied by the emergence of medical care insurance models to pay for costly new technologies, consuming 97 percent of the health dollar in the US and growing at a rate that far exceeded growth in the economy as a whole.

The Ottawa Charter for Health Promotion (1986) ${ }^{1}$ co-sponsored by the World Health Organization defines "health" as "a resource for everyday life, not the objective of living." It is one expression of what is often designated as "positive health." Since health ranges from very poor to very good, one can view the negative dimension of health as diseases and their consequences, while the positive health dimension includes the capacity for living a full and productive life. Public health practitioners have historically concentrated on disease control, that is, the negative dimension of health, because that has constituted the major health problem. Increasingly, however, we are moving into an era in which health itself, not just disease control, has become the goal. While we must still reckon with infectious and chronic diseases, we are also embarking on the third wave of modern public health - extending the quality of life. ${ }^{2}$ Many of the most important strategies for accomplishing this will require improvements in the social, economic and physical determinants of health and well-being. These population-oriented interventions will require actions redolent of the social hygiene movement.

In this paper, we present our view of public health in the US - current population health and health trends, determinants of health, and how we can build an evidence-based public health system during a period of transformation in American health policy. We are unapologetically selective in our choice of examples and emphases. We recognize that many aspects of public health in the US have parallels in other countries and trust that some of our perspectives will resonate with practitioners abroad. 


\section{CONCEPTUALIZATION OF HEALTH}

The World Health Organization defines health as "a state of complete physical, mental and social well-being and not merely the absence of disease or infirmity." ${ }^{3}$ The medical model, which focuses largely on disease and injury and their outcomes, is built upon a pathophysiologic, reductionist approach that dominates Americans' conceptualization of health. Deep understanding of basic biology and a robust market for investments in technological innovations has led to extraordinary medical advances in diagnostics, pharmaceuticals, devices and procedures, which now typify our technology-intensive medical care system. Thus the leading causes of death are traditionally categorized by organ or by disease type: diseases of the heart, cancer, or pneumonia. The medical care system intrinsically focuses on individuals. Even when focused on prevention for risk factor reduction and delivery of clinical preventive services (e.g., hypertension screening, mammography and immunization), the primary goal is reduction in adverse outcomes of diseases.

In contrast, an ecologic or population health model (Figure 1, Table 1) takes a broader perspective and examines the relationship between the innate biologic characteristics of individuals and their interactions with their peer groups, families, communities, schools and workplaces, as well as the broad economic, cultural, social, and physical environmental conditions at the local, national, and global levels. It emphasizes the importance of the social and physical environments that strongly shape patterns of disease and injury as well as our responses to them over the entire life cycle, providing a broader conceptualization of important determinants of health not easily identifiable or rectifiable within the medical model. ${ }^{4}$ Healthy communities, which are defined by having the capacity to allow each individual to be healthy, must address all these components.

The conceptualization of health and its determinants is critically important since it shapes the interventions that we consider and choose. Within the medical model, interventions are focused on specific risk factors or pathophysiologic causes of disease and injury. Hence the use of genomics to predict disease risk, the use of hypertension medications to control blood pressure, and stents and angioplasty to open clogged arteries. The high level of specificity of these procedures facilitates ready assessment through clinical-epidemiological studies. The value of effective technologies can then be assessed through economic evaluation and their use improved through quality improvement strategies. Within the public health framework, on the other hand, interventions are likely to be targeted at policies (e.g., 
clean air laws), the economic, social, and physical environments (e.g., living wage laws, building codes and urban planning, cultural programming), communities (e.g., strengthening community support systems), families (e.g., nurse home visits), or behaviors (e.g., good nutrition), in addition to biologic factors. These interventions generally have broad health impacts (i.e., affect many disease states and overall well-being), as well as important non-health effects. Population health initiatives focus more intensively on interventions at the community and societal levels that have the potential to help the healthy population maximize health, while clinical or medical strategies focus primarily on individuals and strategies for preventing diseases, injuries, and their sequelae.

Table 1

Examples of Determinants of Health within an Ecologic Model

\begin{tabular}{ll}
\hline Biologic factors: & Living and working conditions \\
Genetic characteristics & Employment / living wage \\
Lipid levels & Income \\
& Educational attainment \\
Individual Behaviors & Healthy homes \\
Physical activity & Walkable communities \\
Diet & Transportation systems \\
Tobacco Use & \\
& Broad social, economic, cultural, health \\
Social, Family, and Community & and environmental conditions and \\
Networks & policies at the global, national, state, \\
Social support / Social capital & and local levels \\
Intact families & Climate change \\
Schools & Medical care system \\
& Air pollution \\
& Discrimination and stigma \\
& War, terrorism, natural disaster \\
& Agricultural policy \\
\hline
\end{tabular}

The medical model alone simply cannot create healthy communities, yet the investment in medical care in the US consumes the overwhelming portion of the health dollar. One might ask, "What in the American culture created this situation?" Perhaps it can best be found in the American imagination of the frontier, where rugged individualism became iconic. This was rooted not only in the Constitution and the value of the individual, but also the desire for autonomy and freedom from government intervention. Notions of the common good were evident in religious communities and 
small towns, but in recent years have been more suspect at the national level. Indeed, marketing forces and commercial interests have stoked individual desires at the cost of the common good. This has led to enormous income disparities with over 10 percent of the population living below the poverty level (as compared with 6 percent in France) and faced with associated social and environmental disadvantages, failing schools, broken families, toxic exposures, and fears of violence. The US stresses equality of opportunity but fails to provide an equal right to medical care. In the public health of the future, a better balance will need to be achieved (see Figure 1).

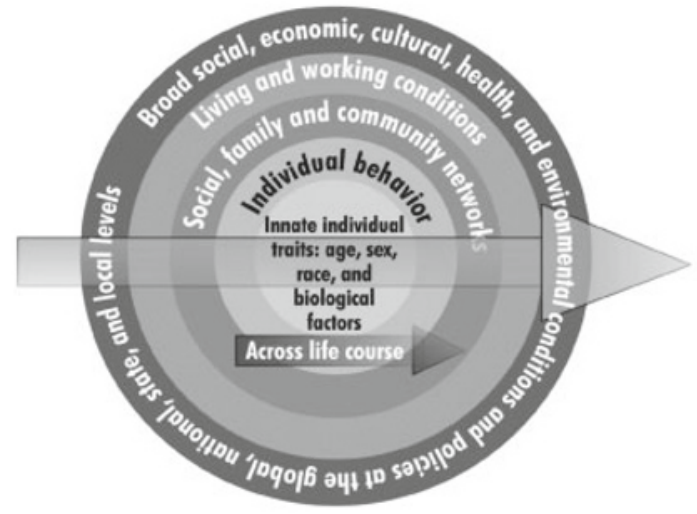

Fig. 1. Ecologic Model of Health. (Adapted from Healthy People 2020).

\section{IMPORTANCE OF A HEALTHY START}

A healthy pregnancy and early childhood can set a child on a healthy life course. We recognize that maternal behaviors and good prenatal care are important. Adequate folic acid intake with cereal grain fortification and supplementation before and during the critical first week of pregnancy reduces neural tube defects by half. Alcohol use during pregnancy causes birth defects and mental retardation. Tobacco use is associated with low birth weight and sudden infant death syndrome. Barker showed that low birth weight babies are at elevated lifelong risk of chronic diseases including heart disease and stroke. ${ }^{5}$ Moreover, maternal stress mediated through maternal cortisol and the pituitary-hypothalamic-adrenal axis contributes to reduced I.Q. ${ }^{6}$

Interventions focused on infant and early childhood development have clearly shown the importance of helping families develop competencies 
that maximize their individual and collective capacity for good health. Protective factors and risk factors early in life can change a person's overall health trajectory. Routine home visits by nurses to pregnant women, and early childhood and family education and support programs, such as Healthy Start and Head Start, are strong examples of interventions that pay dividends over a lifetime. ${ }^{7,8}$

\section{DETERMINANTS OF HEALTH}

\section{Social Environment}

Health is often measured in terms of life expectancy, mortality and infant mortality, and the leading causes of death. Reflecting the medical model of disease, the leading causes of death in the US are heart disease, cancer, and stroke. Foege and McGinnis and later Mokdad reframed the causes of death based on what they called the "actual causes of death" - risk factors such as tobacco, physical activity, nutrition, and alcohol use. ${ }^{9,10}$ This formulation was an important step forward. It forced recognition of the fact that that although a death certificate might say "coronary heart disease," the underlying reasons were sedentary lifestyle, tobacco use, and excessive caloric consumption. However, we can look deeper at not only the root causes of disease and injury - the downsides of health, but also at the factors that improve the quality and value of health - the positive side of health.

These factors, which are the underlying determinants of health, are largely a product of our social and physical environments. Enormous disparities in health outcomes are largely a product of these factors and need to be addressed preferably by solving the problems underlying poor health, as opposed to trying to respond to late biological manifestations of a disease through clinical management.

So what are these factors? They are the very fabric of our societies. Social and economic factors include the family, community, and societal structures. Strong, supportive families and communities foster safe, secure environments and build social capital. Breakdowns in family, neighborhood, and community structure lead to social isolation and violence. ${ }^{11}$ Individuals benefit from receiving a good education and having meaningful employment that provides a living wage. The enormous disparities in education and income are very much a product of unequal opportunities. These disparities are strongly related to variations in health outcomes. ${ }^{12}$ Diversity of communities can be a source of great strength, yet discrimination on 
the basis of age, race, gender, ethnicity, or sexual orientation creates stigmatization and lost opportunity, and translates into challenges to selfefficacy and self-esteem, often resulting in feelings of worthlessness and insufficient capacity to cope with life stresses. It is not surprising that the confluence of these problems fosters environments prone to violence, substance abuse, and lack of hope - elements that destroy the capacity of individuals to live full, rich lives.

In our increasingly complex societies, characterized by great mobility and urbanization, population growth exacerbates demands for scarce resources and jobs. Large families can be difficult to support. Urbanization can lead to depersonalization. Though the US has long since negotiated the demographic transition (rapidly falling mortality followed by rapidly falling birth rates), the population continues to grow through immigration.

Intrinsic to our social environment are governmental and nongovernmental structures and systems - our policies and norms. These structures provide social infrastructure and shape our daily lives. Some are quite specific. Clean air laws and tobacco taxes, for example, have helped modify social norms and reduce cigarette smoking. Agricultural policies directly affect our food supplies. Commodity supports for corn, the largest crop in the US, as well as the presence of inexpensive fructose in high calorie sweetened beverages, have not only affected farmers and agribusiness, but have fostered monoculture, processed foods, and grainfed cattle, all of which contribute to our high-fat, high protein, and high carbohydrate diets. The structure of the medical care system, including regulatory, oversight, and marketing processes for devices, drugs, and diagnostics; insurance, coverage, and reimbursement systems; organization and fragmentation of medical practice; and balance of specialty and primary care, influences the accessibility of care, the type of care that is delivered, the emphasis on technological solutions, and the resulting poor integration in most parts of American medicine.

Although many of the important aforementioned structures and systems have specific biological consequences, their profound influences on overall functioning, attitudes, behaviors, and psychological well-being are not fully understood. Regardless, these factors have impact across the entire spectrum of disease, injury, and well-being. They are apparent not only in manifestations of ill health and well-being, but resilience and resistance as well.

\section{Physical Environment}

Equally important health determinants are the natural and built environments. The natural environment in the US has undergone massive 
change in just a few hundred years. Entire ecosystems, such as prairies, have been virtually eliminated, leading to loss of species and biological diversity. Climate change, largely a product of industrialization and transportation, with anticipated rises in sea level, threatens to inundate coastlines that harbour dense populations. Impending droughts and loss of snow packs in western mountains will disrupt farming and parch many parts of the country. Direct health consequences include changes in patterns of illness due to altered vectors or their environments for disease vectors, and indirect consequences from disruption in society and competition for scarce resources.

Contamination of our air, ground, and water are not only legacies from a bygone age of industrial dumping, but continues to this day as a result of poor sanitary and industrial practices, transportation, agriculture, and energy policies. Toxic exposures occur on account of waste sites and contaminated food supplies, such as mercury-tainted fish, and within occupational settings and homes. Introduction and widespread use of products of uncertain safety, such as plastics made of bisphenol-A, call for reasonable safety measures and the use of good manufacturing practices, and most importantly, the precautionary principle to reduce risk to an absolute minimum. Though some information is commonly available about individual chemical agents, their interactive effects are very poorly established. Endocrine disrupters found in pesticides, dioxin-like compounds, plastics and many everyday products are now widespread and cause endocrine, developmental, reproductive, neurological, and immunological effects in both humans and wildlife. ${ }^{13}$

A community's built environment structures our lives and influences our health. The design of our cities and local streets shapes our ability to incorporate walking into our daily lives and defines our sense of community; our accessibility to recreation, stores, places of worship, employment, and schools; and our safety. Safe homes and buildings reduce injuries and toxic exposures. Public transportation systems enhance accessibility, reduce stress, and promote physical activity, thereby having the potential to counter the obesity epidemic. Parks and recreation areas can be focal points for communities and encourage sports and physical activity. Access to grocery stores and farmers' markets facilitates healthy eating.

\section{BUILDING A HEALTHY COMMUNITY}

A healthy community is one where all sectors contribute to create social and physical environments that foster health. In practice, such a community 
meets basic needs: access to affordable, healthy foods; affordable housing and transportation; and essential services such as medical care and education. It offers a sustainable, healthful environment with clean air and water, open space and parks, low levels of toxic exposures and low emissions, and affordable, sustainable energy. Equally important, it has a constructive economic and social environment with adequate job opportunities, educational opportunities for advancement, and social equity. Last but not least, it offers robust civic and social engagement with safe, supportive families, relationships, homes, and neighborhoods for all parts of society. ${ }^{14}$ Creating the capacity for health is a tall order for public health, particularly since governmental functions and stakeholder expectations are poorly aligned with meeting this need.

\section{CORE CAPACITY OF LOCAL PUBLIC HEALTH AGENCIES IN THE UNITED STATES}

If public health is to create an environment where everyone has the capacity to be healthy and live a full and fulfilling life, it must have the ability to do so. Governmental public health is required to perform ten essential services (Figure 2). These are based on core functions of public health from the IOM report Future of Public Health ${ }^{15}$ and include:

- Assessment - the ability to conduct public health surveillance to measure the health of the population and determinants; investigate health problems and identify causes;

- Policy development - the ability to inform leaders and the general population about health, to develop policy solutions and mobilize support; and

- Assurance - the ability to ensure the health of the population by having a competent workforce to enforce laws; to have medical care available to all; and to evaluate progress as part of a virtuous cycle of quality improvement (Plan/Do/Study/Act).

Unfortunately, many of the 2,794 local health departments in the US ${ }^{16}$ are sparsely staffed and many more are badly under-resourced, so that the ability to deliver those functions is often lacking. Accreditation of public health departments, a process which is being implemented by the newlyformed Public Health Accreditation Board, may lead to consolidation of local health departments and enable an objectively defined core capacity (i.e., the ability to fulfill all the core functions, to be developed in every jurisdiction) (see Figure 2). 


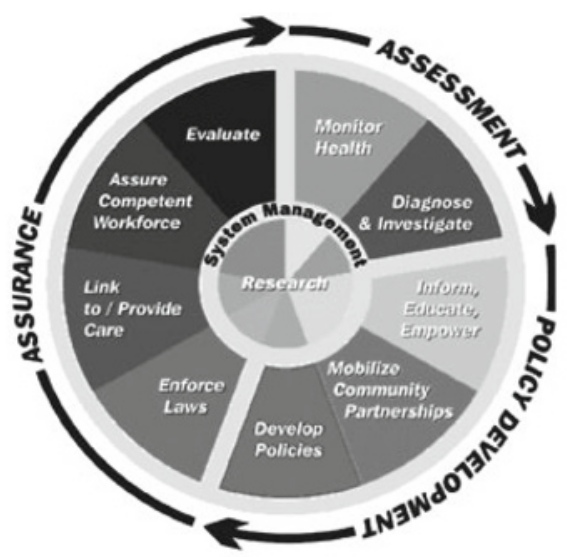

Fig. 2. The Ten Essential Public Health Services.

Source: Ten essential services, Essential Public Health Services Presentation. http://www. cdc.gov/od/ocphp/nphpsp/essentialphservices.htm (Accessed 12 January, 2010).

\section{TRADITIONAL PUBLIC HEALTH PROGRAMS}

At the state and local levels, the specific programs categorized as "public health" vary widely. Some include mental health and alcohol and substance abuse, while others do not. Some have environmental health and maternal and child health components; others do not. Some provide direct medical services; others do not. Despite this variation, there have historically been a number of programs that have been central elements of public health departments. Since the roots of modern public health lie in infectious disease, maternal and child health, and environmental monitoring, it is not surprising that these programs are found in most jurisdictions. These health challenges have by no means disappeared, so these activities remain pillars of the future of public health.

\section{Health Protection}

Though not explicitly enumerated in the ten essential functions, protection of the public's health has taken on new meaning and energy following the terrorist attacks in New York (9/11), as well as Hurricane Katrina in New Orleans. The local public health agency in cooperation with fire, police, and other services manage disaster situations with support and help from non-governmental agencies, as well as state and national authorities. For 
state and local public health departments in jurisdictions that are likely terrorist targets and/or likely sites of natural disasters (e.g., wildfires, earthquakes, hurricanes), this function commands top priority. Health protection in the face of natural and man-made disasters has become more systematized with national policies and preparedness plans. Public health agencies are working in close coordination with law enforcement and protection agencies to address evermore sophisticated threats.

\section{Health Promotion}

Promotion of healthful behaviors has a rich history and has taken the form of health education, regulation of food and housing, planning for safe communities, policy change to discourage unhealthful behaviors and make healthy choices the default option, and community engagement. Energy was initially directed toward control of infectious disease, safety, and improving maternal and child health. As the country underwent the demographic transition and the toll of chronic diseases became apparent, health promotion focused more on individual behavior-associated risk factors contributing to chronic disease, particularly tobacco, hypertension, dyslipidemia, physical inactivity, and poor diet. Nonetheless, obesity and the highly correlated type 2 diabetes have become epidemic. Unlike infectious diseases caused by an identifiable organism with defined modes of transmission, these conditions are a complex product of modern society. For example, the underlying causes of obesity are our sedentary lifestyle combined with overnutrition. Their control requires multifaceted interventions. Changes in public health nutritional interventions, and agricultural and trade policies can improve availability and affordability of fruits and vegetables and reduce sodium in processed foods. Transportation systems, parks, and community designs can encourage walking and physical activity. Educational initiatives, such as menu labelling in restaurants, can reduce caloric intake. Healthy food options can be provided in schools and workplaces.

Because a multifaceted approach is needed to make substantial change, future endeavors are likely to focus on broad policy changes coupled with "place-defined" initiatives. In the latter, a problem is attacked using interventions from multiple sectors, and a variety of synergistic initiatives from many public and non-governmental sectors are brought to bear on a defined population. Obesity initiatives, for example, might include improved master plans, smart growth zoning to shape future development of communities; transportation infrastructure supporting biking, walking, and use of public transportation; construction and maintenance of safe parks; establishing farmer's markets; and walk-to-school programs. 


\section{LEADERSHIP AND ADVOCACY ROLES OF GOVERNMENTAL PUBLIC HEALTH}

The health problems of today and tomorrow are not solvable by governmental public health alone, but governmental public health leadership should play a central and catalytic role. Among the essential public health services, surveillance can paint the picture of health in the community - highlighting strengths and problems, as well as identifying sub-populations at particular risk. As conveners or coordinators, public health officials can bring together key stakeholders to shape a common understanding of the health problems, identify potential solutions, rally support, and coordinate common action. This requires that public health have the necessary infrastructure and capacity, including qualified professionals and information. Today, in the US, resources are provided almost exclusively for categorical programs, such as alcohol rehabilitation or immunizations, diminishing opportunities to address emerging health problems and develop the cross-program and intersectoral solutions required. New funding streams and more flexibility in existing ones are needed to address the most burdensome public health problems and the wide disparities in health among different subpopulations.

\section{EVIDENCE-BASED PRACTICE}

Even with better support of public health initiatives, resources need to be wisely prioritized. Deciding which problems are most important and which interventions to implement presents complex challenges. Although it seems simplistic, we would like to choose actions that have the greatest potential to reduce health burden and build capacity for health. We have traditional ways to measure burden (e.g., mortality, life expectancy, incidence, prevalence, severity, and economic impact), but have fewer general measures to assess the capacity for health. However, models of measuring health burden are only of limited help when applied to non-disease-specific interventions. Calculating the preventable fraction of coronary artery disease mortality from lipid screening and management is straightforward. Performing similar calculations for risk factors like obesity, interventions targeting social or environmental determinants (e.g., reducing income disparities or improving high school graduation rates), or systems changes (e.g., providing universal health insurance) is more complicated and requires other methods, such as health impact assessment. 
Understanding which interventions actually succeed in improving health is critical to effective resource allocation. The U.S. Preventive Services Task Force and the U.S. Community Preventive Services Task Force have made important strides in developing and applying the scientific basis for these assessments. They have characterized interventions and assessed the potential magnitude of effect, the certainty of the information, and the differential effects on populations and subpopulations (based on race, ethnicity, types of communities, income, etc.). The Community Guide ${ }^{17}$ has assessed over two hundred specific population-health interventions and has plans to evaluate hundreds more, as well as update those already completed. These methods are well-suited to the assessment of specific programs and interventions, but are less applicable to intersectoral interventions targeting social and physical environmental determinants and policies. Health impact assessment is another strategy that takes advantage of modelling techniques using the best-available evidence to assess policies and interventions that are not amenable to conventional evaluation techniques. ${ }^{18}$ Assessments can be as local as the health impact of local roadway design or as broad as the health impact of a living wage. First priority should be given to implementing effective and relatively cost-effective interventions, including risk behavior related to HIV, sexually transmitted diseases, and those already discussed to address obesity. If other interventions are used due to a lack of known effective or feasible solutions, they should be carefully evaluated.

\section{SETTING GOALS AND OBJECTIVES}

For over 30 years, the US has used "Healthy People" goals and objectives to provide a national roadmap for health promotion and disease prevention. The current iteration, Healthy People 2020, ${ }^{19}$ links aspirational goals and objectives more strongly to evidence-based practice by providing guidance on priority setting processes and tools, and linking objectives to recommendations of the Guide to Community Preventive Services. ${ }^{17}$ It envisions a society in which all people live long, healthy lives and which seeks to improve health through strengthening policy and practice by:

- Identifying nationwide health improvement priorities;

- Increasing public awareness and understanding of the determinants of health, disease, and disability and the opportunities for progress;

- Providing measurable objectives and goals that can be used to set priorities and monitor progress at the national, state, and local levels;

- Engaging multiple sectors to take actions that are driven by the best available evidence and knowledge; and

- Identifying critical research and data collection needs. 
The Advisory Committee recommends prioritization based on:

1. Overall burden of disease;

2. Preventability or reducibility;

3. Cost-effectiveness (Value);

4. Net health benefit;

5. Synergy of different interventions;

6. Time frame to observe the impact;

7. Potential to reduce health inequities; and

8. Willingness to accept accountability.

These criteria can be applied regardless of the scope of one's responsibilities; whether one is running a highly focused categorical program, such as Chlamydia control, targeting specific health problems, such as prevention of blindness, or has broad responsibility for allocating resources across multiple domains, as do senior public officials. Quality improvement principles require measurement to assess progress and have led to substantial improvement in quality of medical care. ${ }^{20}$ Accountability has been particularly challenging in public health where major initiatives require commitment and action from many stakeholders over extended periods of time. Finding a workable system of accountability and appropriate metrics will be necessary for public health to achieve its full potential.

\section{RESOURCES}

In the US, meeting the medical care needs of the individual invariably seems to take precedence over meeting public health needs. This can be seen in healthcare spending which now consumes over 16 percent of the total gross domestic product, ${ }^{21}$ far more than in any other developed country, and continues to rise far in excess of overall inflation. This trend is simply not sustainable, but efforts to rein in costs have been meager. Openended medical care funding is in stark contrast to public health, where appropriations consistently lag behind need. Many jurisdictions cannot provide even the most basic public health services, let alone have the ability to invest in badly needed information technology infrastructure. Flexible funding streams would allow public health to better address not only specific health problems, but also the underlying social and physical environmental determinants which foster poor health and exacerbate disparities. Movement away from categorical funding for public health programs could restore focus on communities and populations - the real customers of public health - and do so with greater efficiency. 


\section{CONCLUSION}

The next frontier in public health will be to create environments where all can be healthy and achieve their maximum potential. The public will need a modified understanding of what public health is, or what may be termed a New Public Health. Many remain mired in old paradigms of the sanitary era, health education messages, or even think of public health simply as the provider of healthcare services of last resort. We will need a major paradigm shift away from a medical model and even a risk factor model of health to one that recognizes the equally important social and physical environmental determinants. As in other paradigm shifts, the need for medical care and behavior change interventions will not disappear. However, we need to energize public health to take on the interventions and policies that provide the greatest return across the full spectrum of health, not simply in the reduction of disease and injury. Accomplishing these lofty tasks will require strong governmental and intersectoral leadership and collaboration.

Conflicts of interest: None declared.

\section{REFERENCES}

1. Ottawa Charter for Health Promotion. First International Conference on Health Promotion Ottawa, 1986 - WHO/HPR/HEP/95.1. Geneva: World Health Organization; 1986. http://www.who.int/hpr/NPH/docs/ottawa_charter_ hp.pdf (Accessed 5 March, 2010).

2. Breslow L. Perspectives: the third revolution in health. Annu Rev Public Health. 2004;25:xiii-xviii.

3. World Health Organization. Definition of health. Available from URL: http:// www.who.int/about/definition/en/print.html. (Accessed 5 March, 2010).

4. Kindig D, Stoddart G. Models for population health: what is population health? Am J Public Health. 2003;93:380-3.

5. Barker DJP. Commentary: Birthweight and coronary heart disease in a historical cohort. Int J Epidemiol. 2006;35:886-7.

6. LeWinn KZ, Stroud LR, Molnar BE, Ware JH, Koenen KC, Buka SL. Elevated maternal cortisol levels during pregnancy are associated with reduced childhood IQ. Int J Epidemiol. 2009;38:1700-10.

7. Healthy Start [homepage]. Available from URL : http://www.msnbc.msn.com/ id/29959028/ (Accessed 30 September, 2009).

8. National Head Start Foundation [home page]. Available from URL : http:// www.nhsa.org/ (Accessed 30 September, 2009).

9. McGinnis JM, Foege WH. Actual causes of death in the United States. JAMA. 1993;270:2207-12.

10. Mokdad AH, Marks JS, Stroup DF, Gerberding JL. Actual causes of death in the United States, 2000. JAMA. 2004; 291:1238-45. 
11. Putnam RD. Bowling Alone: The collapse and revival of American community. New York: Simon \& Schuster; 2000.

12. Commission to Build a Healthier America. Beyond health care: new directions to a healthier America. Princeton, (NJ): Robert Wood Johnson Foundation; 2009.

13. National Institute for Environmental Health Sciences. Endocrine disruptors. http://www.niehs.nih.gov/health/topics/agents/endocrine/index.cfm (Accessed 12 January 2010).

14. Rudolph L. Climate change: the public health response. California Conference of Local Health Officers, June 2009. State of California. Available from URL: http://www.arb.ca.gov/cc/ab32publichealth/meetings/061809/ dph_climateoverview_061809.pdf (Accessed 5 March, 2010).

15. Institute of Medicine, Committee for the Study of the Future of Public Health. The future of public health. Washington (DC): National Academy Press; 1988.

16. National Association of County and City Health Officials. 2008 national profile of local health departments. Washington (DC): National Association of County and City Health Officials; 2009. Available from URL: http://www.naccho.org/ topics/infrastructure/profile/resources/2008reports/upload/NACCHO_2008_ ProfileReport_post-to-website-2.pdf (Accessed 30 September, 2009).

17. U.S. Task Force on Community Preventive Services. The community guide: what works to promote health. Atlanta: Centers for Disease Control and Prevention. Available from URL: http://www.thecommunityguide.org/index. html (Accessed 12 January, 2010).

18. UCLA School of Public Health. The UCLA Health Impact Assessment Clearinghouse Learning and Information Center (HIA-CLIC). Los Angeles. Available from URL: http://www.ph.ucla.edu/hs/hiaclic/ (Accessed 2 October, 2009).

19. US Department of Health and Human Services. Healthy people 2020: the road ahead. Available from URL: http://www.healthypeople.gov/hp2020/ (Accessed 2 October, 2009).

20. National Committee for Quality Assurance. Washington (DC). Available from URL: http://www.ncqa.org/ (Accessed 2 October, 2009).

21. Centers for Medicare and Medicaid Services. National health expenditure data. Washington (DC): US Department of Health and Human Services. Available from URL: http://www.cms.hhs.gov/nationalhealthexpenddata/ (Accessed 2 October, 2009). 